\title{
Structural basis for branched substrate selectivity in a ketoreductase from Ascaris suum
}

\author{
Hongjun Dong ${ }^{1,4}$ and Michelle C. Y. Chang ${ }^{1,2,3 *}$ \\ ${ }^{1}$ Department of Chemistry, University of California, Berkeley, Berkeley, CA 94720-1460. \\ ${ }^{2}$ Department of Molecular \& Cell Biology, University of California, Berkeley, CA 94720-3200. \\ ${ }^{3}$ Department of Chemical \& Biomolecular Engineering, University of California, Berkeley, CA 94720-1462. \\ ${ }^{4}$ Current Institution: Tianjin Institute of Industrial Biotechnology, Chinese Academy of Sciences, Tianjin, 300308, People's Republic \\ of China \\ *To whom correspondence should be addressed: mcchang@berkeley.edu
}

\section{Materials and Methods}

$\begin{array}{lr}\text { Commercial materials } & \mathrm{S} 2\end{array}$

Bacterial strains $\quad S 2$

Plasmid construction

Expression and purification of proteins of AsHadh2 variants $\quad$ S3

Crystallization and determination of the AsHadh2-NAD+crystal structure S4

Docking of OMB-COA to the AsHadh2-NAD+crystal structure S4

AsHadh2 library preparation and in vivo screening.

Steady-state kinetic characterization of AsHadh2 variants $\quad S 5$

Molecular dynamics simulations $\quad$ S6

\section{Supplementary Data}

Table S1. Strains, plasmids, and primers $\quad S 7$

Table S2. Data collection and refinement statistics for the AsHadh2-NAD+ structure S10

Figure S1. Crystal structure of AsHadh2 S11

Figure S2. AsHadh2 bound to $N A D^{+} \quad S 12$

Figure S3. Docking of (S)-3-oxo-2-methylbutyryl-CoA into AsHadh2-NAD+ S13

Figure S4. Generation and screening of an AsHadh2 pocket library S14

Figure S5. SDS-PAGE of purified Hadh2 variants $\quad S 15$

Figure S6. Steady-state kinetic analysis for Hadh2 variants with OMB-CoA and OB-CoA S16

Figure S7. Molecular dynamics simulations of AsHadh2

References $\quad$ S18 


\section{Materials and Methods}

Commercial materials. Luria-Bertani (LB) broth/agar, Terrific Broth (TB), and chloramphenicol $(\mathrm{Cm})$ were purchased from EMD Biosciences (Darmstadt, Germany). Lysozyme, 2-mercaptoethanol (BME), bovine serum albumin (BSA), acetoacetyl coenzyme A (3-oxobutyryl-CoA, OB-CoA), nicotinamide adenine dinucleotide reduced form dipotassium salt $(\mathrm{NADH})$, nicotinamide adenine dinucleotide hydrate $\left(\mathrm{NAD}^{+}\right)$, formic acid, adipic acid, sodium 3-hydroxybutyrate, thiophenol, 4-dimethylaminopyridine (DMAP), ethyl 3-methyl-2oxobutyrate, and N,N'-dicyclohexylcarbodiimide (DCC) were purchased from Sigma-Aldrich (St. Louis, MO). 2-Methyl-3-hydroxybutyric acid was purchased from Santa Cruz Biotechnology (Dallas, Texas). Carbenicillin (Cb), kanamycin (Km), isopropyl- $\beta$-Dthiogalactopyranoside (IPTG), dithiothreitol (DTT), 4-(2-hydroxyethyl)-1piperazineethanesulfonic acid (HEPES), acetonitrile, and EMD Millipore MultiScreenHTS 96Well Filter Plates were purchased from Fisher Scientific (Pittsburgh, PA). Rectangular 24-deep well plates were purchased from VWR (Radnor, PA). Aeraseal Sealing Film was purchased from Research Products International (Mount Prospect, IL). Restriction enzymes and Phusion DNA polymerase were purchased from NEB (Ipswich, MA). DNA purification kits and NiNTA agarose were purchased from Qiagen (Valencia, CA). Oligonucleotides were purchased from IDT (Coralville, IA), resuspended at a stock concentration of $100 \mu \mathrm{M}$ in water, and stored at $-20{ }^{\circ} \mathrm{C}$ for long-term storage. dNTPs were purchased from Invitrogen (Carlsbad, CA). Acrylamide/bis-acrylamide (30\%, 37.5:1), electrophoresis grade sodium dodecyl sulfate (SDS), N,N,N',N'-tetramethyl-ethane-1,2-diamine (TEMED), and ammonium persulfate, and Bio-Rad Protein Assay reagent were purchased from Bio-Rad (Hercules, CA). Imidazole was purchased from Acros Organics (Morris Plains, NJ). cOmplete EDTA-free protease inhibitor was purchased from Roche (Penzberg, Germany). Amicon Ultraspin concentrators were purchased from Merck Millipore (Cork, Ireland). Tris(2-carboxyethyl)phosphine (TCEP) was purchased from Biosynth, Inc. (Itasca, IL). PageRuler ${ }^{\mathrm{TM}}$ Plus prestained protein ladder was purchased from Fermentas (Glen Burnie, Maryland).

Bacterial strains. The $\mathrm{T} 1$ bacteriophage-resistant strain E. coli $\mathrm{DH} 10 \mathrm{~B}^{\mathrm{T} 1 \mathrm{R}}$ was used for plasmid construction. E. coli BAP1 was used for in vivo screening of the AsHadh2 library and E. coli BL21(DE3) ${ }^{\mathrm{T} 1 \mathrm{R}}$ was used for heterologous protein production. 
Plasmid construction. Plasmid construction was carried out using standard molecular biology techniques and the Gibson protocol [1]. PCR amplifications were carried out with Phusion DNA polymerase, following manufacturer instructions. Primer sequences are listed in Table S1. Constructs were verified by sequencing.

AsHadh2 expression vectors: The AsHadh2 expression vector pET23a-AsHadh2-PreSC was constructed by inverse PCR of pET23a-AsHadh2 (constructed by Dr. Michael R. Blaisse) using primers 156-PreSC-AsHadh2-R and 157-PreSC-AsHadh2-F, to generate pET23aAsHadh2-PreSC containing AsHadh2 with a N-terminal PreScission protease site and His6 tag. The mutations identified from the in vivo screen were introduced into pET23a-AsHadh2PreSC by same inverse PCR strategy using primers 461-468 (see primer table).

AsHadh2 libraries: The plasmid 1 pAsAcat3-AsHadh2 [2] was used as template for reverse PCR using primers Hadh-X-A and Hadh-X-B primers (see primer table) for each site saturation mutation.

Expression and purification of $\mathrm{His}_{6}$-AsHadh2 variants. TB (1L) containing carbenicillin $(50 \mu \mathrm{g} / \mathrm{mL})$ in a $2.5 \mathrm{~L}$ Ultra yield baffled shake flasks (Thomson Instrument Company, Oceanside, $\mathrm{CA}$ ) was inoculated to $\mathrm{OD}_{600}=0.05$ with an overnight $\mathrm{TB}$ culture of freshlytransformed $E$. coli $\mathrm{BL} 21(\mathrm{DE} 3)^{\mathrm{T} 1 \mathrm{R}}$ containing the expression plasmid. The cultures were grown at $37^{\circ} \mathrm{C}$ at $200 \mathrm{rpm}$ to $\mathrm{OD}_{600}=0.6$ to 0.8 at which point cultures were cooled on ice for $20 \mathrm{~min}$, followed by induction of protein expression with IPTG $(0.4 \mathrm{mM})$, and overnight growth at $16^{\circ} \mathrm{C}$. Cell pellets were harvested by centrifugation at $8,000 \times g$ for $5 \mathrm{~min}$ at $4^{\circ} \mathrm{C}$ and stored at $-80^{\circ} \mathrm{C}$ for protein purification.

Frozen cell pellets were thawed and resuspended at $3 \mathrm{~mL}$ per gram cell paste with Wash Buffer (50 mM HEPES, $10 \mathrm{mM}$ imidazole, $300 \mathrm{mM} \mathrm{NaCl}, 5 \% v / v$ glycerol, $\mathrm{pH}$ 7.5) supplemented with $8 \mathrm{mM}$ BME, $1 \mathrm{mg} / \mathrm{mL}$ lysozyme, $0.04 \mu \mathrm{L} / \mathrm{ml}$ benzonase, and one cOmplete EDTA-free protease inhibitor cocktail tablet per $25 \mathrm{~mL}$. The cells were lysed by sonication using a Misonix Sonicator 3000, and lysate was centrifuged at $10,000 \times g$ for $30 \mathrm{~min}$ at $4{ }^{\circ} \mathrm{C}$ to separate soluble and insoluble fractions. The soluble lysate was loaded onto Ni-NTA resin column (Qiagen, $1.5 \mathrm{~mL}, 50 \%$ slurry in 30\% ethanol) by gravity flow. The column was washed with $40 \mathrm{~mL}$ Wash Buffer with $5 \mathrm{mM}$ BME and $50 \mathrm{~mL}$ of 90\% Wash Buffer: 10\% Elution Buffer (50 mM HEPES, $250 \mathrm{mM}$ imidazole, $300 \mathrm{mM} \mathrm{NaCl}, 5 \%$ glycerol, $\mathrm{pH}$ 7.5) with $5 \mathrm{mM}$ BME. 
Protein was eluted with $10 \mathrm{~mL}$ Elution Buffer containing $5 \mathrm{mM}$ BME. Fractions containing the target protein were pooled by $\mathrm{A}_{280 \mathrm{~nm}}$ and concentrated using a $10 \mathrm{kDa}$ Amicon Ultraspin concentrator. Finally, protein was exchanged into Storage Buffer (20 mM HEPES, $100 \mathrm{mM}$ $\mathrm{NaCl}, 10 \% v / v$ glycerol, $\mathrm{pH} 7.5)$ with $0.2 \mathrm{mM}$ TCEP using a $10 \mathrm{kDa}$ Amicon Ultraspin concentrator. Protein concentration was estimated using the Bio-Rad Bradford Protein Assay Kit using BSA as standard. Aliquots were frozen in liquid nitrogen and stored at $-80^{\circ} \mathrm{C}$.

Crystallization and determination of the $\mathrm{His}_{6}-\mathrm{AsHadh}_{2}-\mathrm{NAD}^{+}$crystal structure. Crystals of His6-AsHadh2-NAD ${ }^{+}$complex were obtained by hanging drop vapor diffusion by combining $800 \mathrm{~nL}$ of protein solution $\left(3 \mathrm{mg} / \mathrm{mL}\right.$ AsHadh2, $\left.1 \mathrm{mM} \mathrm{NAD}{ }^{+}\right)$and reservoir solution $(35 \%$ PEG550 MME, $144 \mathrm{mM}$ potassium sodium tartrate, $100 \mathrm{mM}$ MES, pH6.5 $2 \mathrm{M}$ sodium malonate, pH 6.5). Crystals were observed within $24 \mathrm{~h}$, and crystals were fished out with a loop and flashfrozen by immersion in liquid nitrogen. Data were collected at Beamline 8.3.1 at the Advanced Light Source (Lawrence Berkeley National Laboratory). Data were processed with XDS [3] and scaled and merged with Aimless [4]. Molecular replacement using BALBES [5] was used for phase determination. The molecular replacement model was one monomer of rat brain type II 3-hydroxyacyl-CoA dehydrogenase ( $\mathrm{rHadh} 2,1 \mathrm{E} 3 \mathrm{~S})$ based on sequence alignment with AsHadh2. Any missing parts of the model were manually built using WinCoot [0]. Iterative cycles using Phenix.refine [7] and manual refinement in WinCoot were used to generate the final model, with Phenix.refine strategies based on XYZ coordinates, real-space refinement, atomic occupancies, and individual B-factors. Chimera [8] was used for visualization of final structures. The data statistics are summarized in Table S2. The refined His6-AsHadh2-NAD ${ }^{+}$ complex models and structure factors were deposited in the Protein Data Bank as the PDB ID 7N09.

Docking of OMB-CoA to the His ${ }_{6}-$ AsHadh2-NAD $^{+}$crystal structure. Docking of OMBCoA to His6-AsHadh2-NAD ${ }^{+}$was performed using Glide SP $[9,10]$ in the Maestro molecular visualization environment developed by SCHRODINGER (http://www.schrodinger.com). The OMB-CoA ligand was generated from acetoacetyl-coenzyme A (CAA) ligand from PDB database by adding one $(S)$-methyl group at the $\mathrm{C} 2$ of acetoacetyl moiety. The chain A structure of His6-AsHadh2-NAD ${ }^{+}$complex was processed by Protein Preparation Wizard of Maestro for docking. To define the grid for docking, the chain A was aligned with RePhaB-NADP ${ }^{+}-\mathrm{CAA}$ (PDB ID: 3VZS), and the space occupied by CAA was set as the receptor grid. Finally, OMB- 
CoA was docked as a flexible ligand into prepared AsHadh2 by Glide program, and top eight poses were selected for further analysis.

AsHadh2 library transformation and in vivo screening. E. coli BAP1 was transformed with pTrc33-TesB and pAsAcat3-AsHadh2* generated by NNK saturation mutagenesis. 24 colonies were picked for each positional library for a total of 336 variants. Strains were cultured in 24-deep well plates for $2 \mathrm{~d}$ at $30^{\circ} \mathrm{C}$ in TB broth containing $2 \%(w / v)$ glucose, $0.2 \%(w / v)$ sodium propionate, and $0.418 \%(w / v)$ MOPS. 3-Hydroxyacids from cell culture were quantified by liquid chromatography-triple quadrupole mass spectrometry as previously reported [2]. The sum of the $\%$ increase in $\mathrm{HMB}$ and $\%$ decrease in $\mathrm{HB}(\Delta \mathrm{HMB} \%-\Delta \mathrm{HB} \%)$ was used to variant selectivity compared to wild-type with $\triangle \mathrm{HB} \%$ or $\Delta \mathrm{HMB} \%=\frac{\text { titer of mutant - titer of wildtype }}{\text { titer of wildtype }}$. The AsHadh2 variants corresponding to higher selectivity for either HMB or HB were regrown for DNA sequencing. When the site of mutation was identified, $\sim 100$ colonies were picked and sequenced for positional library of interest in order to obtain maximal portion of 19 variants at that position. The missed mutants were generated by inverse PCR using primers 292-328 (see primer table). These saturation libraries for I55, M205, P209, and R213 were then analyzed similarly.

Steady-state kinetic characterization of AsHadh2 variants. AsHadh2 variants were assayed by monitoring the initial consumption of NADPH at $340 \mathrm{~nm}$ on a Beckman DU-800 spectrophotometer (Beckman, USA). Assays were performed at room temperature in a $1 \mathrm{~cm}-$ pathlength quartz cuvette in a total volume of $100 \mu \mathrm{L}$ in HEPES buffer (10 mM HEPES, 150 $\left.\mathrm{mM} \mathrm{KCl}, 10 \mathrm{mM} \mathrm{KH}_{2} \mathrm{PO}_{4}, \mathrm{pH} 7.5\right)$ with $\mathrm{NADH}(0.15 \mathrm{mM})$, varying amounts of 3-oxoacylCoA substrate (either OB-CoA or OMB-CoA), and enzyme. Synthetic 3-oxo-2-methylbutyrylCoA was prepared and quantified as previously reported[2]. The reaction was initiated by addition of 3-oxoacyl-CoA substrate and mixing rapidly by pipette. The concentrations of AsHadh2 for OB-CoA and OMB-CoA are listed.

\begin{tabular}{ccc}
\hline $\begin{array}{c}\text { AsHadh2 } \\
\text { (nM) }\end{array}$ & OB-CoA & OMB-CoA \\
\hline WT & 200 & 40 \\
I155A & 696 & 40 \\
I155T & 682 & 40 \\
M205F & 136 & 40 \\
R213S & 68 & 40 \\
R213L & 140 & 40 \\
\hline
\end{tabular}


The data were fit to different equations according to the performance of each enzyme: I155T/OB-CoA was fit to standard Michaelis-Menten equation (Eq. 1):

$$
v_{0}=\frac{V_{\max }[S]_{0}}{K_{M}+[S]_{0}}
$$

Eq. 1

Wild type/OB-CoA, I155A/OB-CoA, M205F/OB-CoA, R213L/OB-CoA, R213S/OB-CoA, I155A/OMB-CoA, and I155T/OMB-CoA were fit to a modified Michaelis-Menten equation with substrate inhibition (Eq. 2):

$$
v_{0}=\frac{V_{\max }[S]_{0}}{K_{M}+[S]_{0}\left(1+\frac{[S]_{0}}{K_{I}^{n}}\right)}
$$

The remaining combinations that could not be fit well to either of these equations were fit to the Hill equation without (Eq. 3, wild type/OMB-CoA and M205F/OMB-CoA) or with (Eq. 4, R213L/OMB-CoA and R213S/OMB-CoA) substrate inhibition:

$$
\begin{aligned}
& v_{0}=\frac{V_{\max }[S]_{0}^{n}}{K_{M}^{n}+[S]_{0}^{n}} \\
& v_{0}=\frac{V_{\max }[S]_{0}^{n}}{K_{M}^{n}+[S]_{0}^{n}\left(1+\frac{[S]_{0}^{n}}{K_{I}^{n}}\right)}
\end{aligned}
$$

Molecular dynamics simulations. The initial coordinate used for molecular dynamics simulations was the OMB-CoA docked structure of AsHadh2-NADH. The complex structure of AsHadh2-NADH-OMB-CoA was also used to generate AsHadh2-NADH-OB-CoA structure by deleting the alpha-methyl group of acyl moiety of OMB-CoA. The initial unit cells were built by using the SPC solvent model with the OPLS3e force field in the system builder panel of Desmond (Schrödinger Release 2019-3). For molecular dynamics simulation, the total simulation time was set to $50 \mathrm{~ns}$, with 5 ps trajectory recording intervals, to reach the equilibrium system. The system energy was set to 1.2 , and the ensemble class used was NPT. Simulations were set to run at $300.0 \mathrm{~K}$ and at 1.01325 bar. 


\section{Supplementary Data}

Table S1. Strains, plasmids, and primers used in this study. (A) Strains and plasmids. (B) Primers.

\section{A. Strains and plasmids}

\begin{tabular}{|c|c|c|}
\hline Strain & Genotype & Source \\
\hline $\mathrm{DH} 10 \mathrm{~B}^{\mathrm{T} 1 \mathrm{R}}$ & $\begin{array}{l}\mathrm{F}^{-} \text {endA1 recA1 galE15 galK16 nupG rpsL fhuA } \Delta / a c X 74 \\
\text { \$80d/acZ } \Delta \mathrm{M} 15 \text { araD139 } \Delta(\text { ara,leu }) 7697 \text { mcrA } \Delta(\text { mrr- } \\
\text { hsdRMS-mcrBC) tonA }\end{array}$ & Invitrogen \\
\hline $\mathrm{BL} 21(\mathrm{DE} 3)^{\mathrm{T} 1 \mathrm{R}}$ & $\begin{array}{l}\mathrm{F}^{-} \text {omp T gal dcm lon hsd } \mathrm{S}_{\mathrm{B}}\left(\mathrm{r}_{\mathrm{B}^{-}} \mathrm{m}_{\mathrm{B}}^{-}\right) \lambda(\mathrm{DE} 3 \text { [lacl lacUV5-T7 } \\
\text { gene } 1 \text { ind1 sam7 nin5]) tonA }\end{array}$ & Novagen \\
\hline BAP1 & $\begin{array}{l}\mathrm{F}^{-} \text {ompT gal dcm lon hsdS }{ }_{\mathrm{B}}\left(\mathrm{r}_{\mathrm{B}}^{-} \mathrm{m}_{\mathrm{B}}^{-}\right) \lambda(\mathrm{DE} 3 \text { [lacl lacUV5-T7 } \\
\text { gene } 1 \text { ind1 sam7 nin5]) } \Delta p r p R B C D E(\text { sfp (T7), prpE } \\
\text { (T7))] }\end{array}$ & Ref. 11 \\
\hline Plasmid & Description & Source \\
\hline pET23a & $\mathrm{T} 7, \mathrm{lacl}, \mathrm{Cb}^{\mathrm{r}}, \mathrm{pBR} 322$ & Novagen \\
\hline pET23a-AsHadh2 & His10-Xa-AsHadh (T7), lacl, Cbr, pBR322 & M. Blaisse \\
\hline pET23a-AsHadh2-PreSC & AsHadh2-PreSC-His 10 (T7), lacl, Cbr, pBR322 & This study \\
\hline pET23a-AsHadh2 I155A-PreSC & AsHadh2 I155A-PreSC-His10 (T7), lacl, Cbr , pBR322 & This study \\
\hline pET23a-AsHadh2 I155T-PreSC & AsHadh2 I155T-PreSC-His10 (T7), lacl, Cbr , pBR322 & This study \\
\hline pET23a-AsHadh2 M205F-PreSC & AsHadh2 M205F-PreSC-His 10 (T7), lacl, Cb', pBR322 & This study \\
\hline pET23a-AsHadh2 R213S-PreSC & AsHadh2 R213S-PreSC-His10 (T7), lacl, Cbr, pBR322 & This study \\
\hline pET23a-AsHadh2 R213L-PreSC & AsHadh2 R213L-PreSC-His10 (T7), lacl, Cbr, pBR322 & This study \\
\hline pAsAcat3-AsHadh2 & AsAcat3-AsHadh2 (Trc), lacl, $\mathrm{Cb}^{\mathrm{r}}$, pBR322 & Ref. 2 \\
\hline pAsAcat3-AsHadh2 library & AsAcat3-AsHadh2 variant (Trc), lacl, $\mathrm{Cb}^{\mathrm{r}}$, pBR322 & This study \\
\hline pTrc33-TesB & $\operatorname{TesB}(9.2 \mathrm{k})$ (Trc), $\mathrm{Cm}^{\mathrm{r}}, \mathrm{p} 15 \mathrm{a}$ & Ref. 12 \\
\hline
\end{tabular}

\section{B. Primers}

\begin{tabular}{ll}
\hline Primer & Sequence \\
\hline 156-PreSC-AsHadh2-R & CGGGCCCTGAAAAAGCACTTCTAGATGATGATGATGATGATGGCTGCTAGCCATATG \\
157-PreSC-AsHadh2-F & CTAGAAGTGCTTTTTCAGGGCCCGTCCGCACTGCGCTCCACGAAAGGTCTGGTCGCGCTG \\
206-Hadh-A94-A & GCGCGGGTATTNNKTACAGCTTTAAACTGTTCAACGTGAAGAAGAAAAAA \\
207-Hadh-A94-B & TTAAAGCTGTAMNNAATACCCGCGCAGTTCACCGCAACGTCCAAACGACC \\
210-Hadh-A153-A & TTATTAATACCNNKAGCATTGCAGCGTTCGACGGTCAAGCCGGCCAAAGC \\
211-Hadh-A153-B & GCTGCAATGCTMNNGGTATTAATAATAACACCGCGTTGGCCCATCTCGTC \\
214-Hadh-I155-A & ATACCGCAAGCNNKGCAGCGTTCGACGGTCAAGCCGGCCAAAGCGCGTAT \\
\hline
\end{tabular}




\begin{tabular}{|c|c|}
\hline 215-Hadh-I155-B & TCGAACGCTGCMNNGCTTGCGGTATTAATAATAACACCGCGTTGGCCCAT \\
\hline 216-Hadh-A156-A & CCGCAAGCATTNNKGCGTTCGACGGTCAAGCCGGCCAAAGCGCGTATAGC \\
\hline 217-Hadh-A156-B & CCGTCGAACGCMNNAATGCTTGCGGTATTAATAATAACACCGCGTTGGCC \\
\hline 218-Hadh-Q164-A & GTCAAGCCGGCNNKAGCGCGTATAGCGCCTCCAAGGGCGCGATTGTTGGC \\
\hline 219-Hadh-Q164-B & CTATACGCGCTMNNGCCGGCTTGACCGTCGAACGCTGCAATGCTTGCGGT \\
\hline 226-Hadh-P197-A & TGACCATCGCTNNKGGCATCTTTGACACCCCGATGATGGCGTCCTTCCCG \\
\hline 227-Hadh-P197-B & TCAAAGATGCCMNNAGCGATGGTCACAACGCGGATGCCATCGTCGGCGAA \\
\hline 228-Hadh-G198-A & CCATCGCTCCGNNKATCTTTGACACCCCGATGATGGCGTCCTTCCCGGAC \\
\hline 229-Hadh-G198-B & GTGTCAAAGATMNNCGGAGCGATGGTCACAACGCGGATGCCATCGTCGGC \\
\hline 230-Hadh-I199-A & TCGCTCCGGGCNNKTTTGACACCCCGATGATGGCGTCCTTCCCGGACAAA \\
\hline 231-Hadh-I199-B & GGGGTGTCAAAMNNGCCCGGAGCGATGGTCACAACGCGGATGCCATCGTC \\
\hline 232-Hadh-F200-A & CTCCGGGCATCNNKGACACCCCGATGATGGCGTCCTTCCCGGACAAAGTT \\
\hline 233-Hadh-F200-B & ATCGGGGTGTCMNNGATGCCCGGAGCGATGGTCACAACGCGGATGCCATC \\
\hline 238-Hadh-M204-A & TTGACACCCCGNNKATGGCGTCCTTCCCGGACAAAGTTCGTAACTTCCTG \\
\hline 239-Hadh-M204-B & AAGGACGCCATMNNCGGGGTGTCAAAGATGCCCGGAGCGATGGTCACAAC \\
\hline 240-Hadh-M205-A & ACACCCCGATGNNKGCGTCCTTCCCGGACAAAGTTCGTAACTTCCTGATT \\
\hline 241-Hadh-M205-B & GGGAAGGACGCMNNCATCGGGGTGTCAAAGATGCCCGGAGCGATGGTCAC \\
\hline 244-Hadh-F208-A & TGATGGCGTCCNNKCCGGACAAAGTTCGTAACTTCCTGATTGGTTTGGTG \\
\hline 245-Hadh-F208-B & ACTTTGTCCGGMNNGGACGCCATCATCGGGGTGTCAAAGATGCCCGGAGC \\
\hline 246-Hadh-P209-A & TGGCGTCCTTCNNKGACAAAGTTCGTAACTTCCTGATTGGTTTGGTGCCG \\
\hline 247-Hadh-P209-B & CGAACTTTGTCMNNGAAGGACGCCATCATCGGGGTGTCAAAGATGCCCGG \\
\hline 248-Hadh-R213-A & CGGACAAAGTTNNKAACTTCCTGATTGGTTTGGTGCCGAATCCGAAGCGT \\
\hline 249-Hadh-R213-B & ATCAGGAAGTTMNNAACTTTGTCCGGGAAGGACGCCATCATCGGGGTGTC \\
\hline 292-R213E-F & CGGACAAAGTTGAAAACTTCCTGATTGGTTTGGTGCCGAATCCGAAGCGT \\
\hline 293-R213E-R & ATCAGGAAGTTTTCAACTTTGTCCGGGAAGGACGCCATCATCGGGGTGTC \\
\hline 294-I155D-F & ATACCGCAAGCGATGCAGCGTTCGACGGTCAAGCCGGCCAAAGCGCGTAT \\
\hline 295-I155D-R & TCGAACGCTGCATCGCTTGCGGTATTAATAATAACACCGCGTTGGCCCAT \\
\hline 296-I155G-F & ATACCGCAAGCGGCGCAGCGTTCGACGGTCAAGCCGGCCAAAGCGCGTAT \\
\hline 297-I155G-R & TCGAACGCTGCGCCGCTTGCGGTATTAATAATAACACCGCGTTGGCCCAT \\
\hline 298-I155V-F & ATACCGCAAGCGTGGCAGCGTTCGACGGTCAAGCCGGCCAAAGCGCGTAT \\
\hline 299-I155V-R & TCGAACGCTGCCACGCTTGCGGTATTAATAATAACACCGCGTTGGCCCAT \\
\hline $300-I 155 W-F$ & ATACCGCAAGCTGGGCAGCGTTCGACGGTCAAGCCGGCCAAAGCGCGTAT \\
\hline $301-I 155 W-R$ & TCGAACGCTGCCCAGCTTGCGGTATTAATAATAACACCGCGTTGGCCCAT \\
\hline 302-M205E-F & ACACCCCGATGGAAGCGTCCTTCCCGGACAAAGTTCGTAACTTCCTGATT \\
\hline 303-M205E-R & GGGAAGGACGCTTCCATCGGGGTGTCAAAGATGCCCGGAGCGATGGTCAC \\
\hline $304-M 205 N-F$ & ACACCCCGATGAACGCGTCCTTCCCGGACAAAGTTCGTAACTTCCTGATT \\
\hline $305-M 205 N-R$ & GGGAAGGACGCGTTCATCGGGGTGTCAAAGATGCCCGGAGCGATGGTCAC \\
\hline 306-M205Y-F & ACACCCCGATGTATGCGTCCTTCCCGGACAAAGTTCGTAACTTCCTGATT \\
\hline 307-M205Y-R & GGGAAGGACGCATACATCGGGGTGTCAAAGATGCCCGGAGCGATGGTCAC \\
\hline 308-P209D-F & TGGCGTCCTTCGATGACAAAGTTCGTAACTTCCTGATTGGTTTGGTGCCG \\
\hline 309-P209D-R & CGAACTTTGTCATCGAAGGACGCCATCATCGGGGTGTCAAAGATGCCCGG \\
\hline 310-P209I-F & TGGCGTCCTTCATTGACAAAGTTCGTAACTTCCTGATTGGTTTGGTGCCG \\
\hline 311-P209I-R & CGAACTTTGTCAATGAAGGACGCCATCATCGGGGTGTCAAAGATGCCCGG \\
\hline 312-P209N-F & TGGCGTCCTTCAACGACAAAGTTCGTAACTTCCTGATTGGTTTGGTGCCG \\
\hline $313-P 209 N-R$ & CGAACTTTGTCGTTGAAGGACGCCATCATCGGGGTGTCAAAGATGCCCGG \\
\hline 314-R213E-F & CCTTCCCGGACAAAGTTGAAAACTTCCTGATTGGTTTG \\
\hline 315-R213 & AACTTTGTCCGGGAAGGACGCCATCATCG \\
\hline 316-I155D-F & TGTTATTATTAATACCGCAAGCGATGCAGCGTTCGACG \\
\hline
\end{tabular}




\begin{tabular}{ll}
\hline 317-I155 & GCTTGCGGTATTAATAATACACCGCGTTGGCC \\
318-I155G-F & TGTTATTATTAATACCGCAAGCGGCGCAGCGTTCGACG \\
319-I155V-F & TGTTATTATTAATACCGCAAGCGTGGCAGCGTTCGACG \\
320-I155W-F & TGTTATTATTAATACCGCAAGCTGGGCAGCGTTCGACG \\
321-M205E-F & CTTTGACACCCCGATGGAAGCGTCCTTCC \\
322-M205 & CATCGGGGTGTCAAAGATGCCCGGAGC \\
323-M205N-F & CTTTGACACCCCGATGAACGCGTCCTTCC \\
324-M205Y-F & CTTTGACACCCCGATGTATGCGTCCTTCC \\
325-P209D-F & GATGATGGCGTCCTTCGATGACAAAGTTCG \\
326-P209 & GAAGGACGCCATCATCGGGGTGTCAAAGA \\
327-P209I-F & GATGATGGCGTCCTTCATTGACAAAGTTCG \\
328-P209N-F & GATGATGGCGTCCTTCAACGACAAAGTTCG \\
461-I155A-P1 & GTGTTATTATTAATACCGCAAGCGCGGCAGCGTTCGACG \\
462-I155T-P1 & GTGTTATTATTAATACCGCAAGCACCGCAGCGTTCGACG \\
463-M205F-P1 & TCTTTGACACCCCGATGTTTGCGTCCTT \\
464-R213S-P1 & CCTTCCCGGACAAAGTTAGCAACTTCCTGATTGG \\
465-R213L-P1 & CCTTCCCGGACAAAGTTCTGAACTTCCTGATTGG \\
466-I155-P2 & GCTTGCGGTATTAATAAAACACCGCGTTGGCC \\
467-M205-P2 & CATCGGGGTGCAAAGATGCCCGGAGC \\
468-R213-P2 & AACTTTGTCCGGGAAGGACGCCATCATCG \\
\hline
\end{tabular}


Table S2. Data collection and refinement statistics for the AsHadh2-NAD ${ }^{+}$structure.

\begin{tabular}{|c|c|}
\hline & AsHadh2, NAD ${ }^{+}$bound \\
\hline \multicolumn{2}{|l|}{ Data collection } \\
\hline Wavelength $(\AA)$ & 1.116 \\
\hline Space group & C 121 \\
\hline \multicolumn{2}{|l|}{ Unit cell } \\
\hline$a, b, c(\AA)$ & 128.554 .989 .3 \\
\hline$\alpha, \beta, \gamma\left(^{\circ}\right)$ & 9013190 \\
\hline Resolution $(\AA)$ & $64.050-2.0(2.072-2.0)^{*}$ \\
\hline Total reflections & $63882(6307)$ \\
\hline Unique reflections & $31971(3160)$ \\
\hline Multiplicity & $2.0(2.0)$ \\
\hline Completeness (\%) & $99.86(99.15)$ \\
\hline Mean I/sigma(I) & $12.53(1.95)$ \\
\hline Wilson B-factor & 31.22 \\
\hline$R_{\text {merge }}$ & $0.02678(0.3468)$ \\
\hline$R_{\text {meas }}$ & $0.03787(0.4905)$ \\
\hline $\mathrm{R}_{\mathrm{pim}}$ & $0.02678(0.3468)$ \\
\hline $\mathrm{CC}_{1 / 2}$ & $0.999(0.81)$ \\
\hline $\mathrm{CC}^{*}$ & $1(0.946)$ \\
\hline \multicolumn{2}{|l|}{ Refinement } \\
\hline Resolution $(\AA)$ & $64.050-2.0$ \\
\hline Reflections used in refinement & 31959 (3160) \\
\hline Reflections used for $R_{\text {free }}$ & $1448(148)$ \\
\hline $\mathrm{R}_{\text {work }} / \mathrm{R}_{\text {free }}$ & $0.1768(0.2896) / 0.2191(0.3350)$ \\
\hline $\mathrm{CC}_{\text {work }} / \mathrm{CC}_{\text {free }}$ & $0.967(0.854) / 0.973(0.722)$ \\
\hline Number of non-hydrogen atoms & 4261 \\
\hline Macromolecules & 3798 \\
\hline Ligands & 88 \\
\hline Solvent & 375 \\
\hline Protein residues & 518 \\
\hline \multicolumn{2}{|l|}{ RMSD } \\
\hline Bond lengths $(\AA)$ & 0.007 \\
\hline Bond angles $\left({ }^{\circ}\right)$ & 0.95 \\
\hline \multicolumn{2}{|l|}{ Ramachandran } \\
\hline Favored (\%) & 96.89 \\
\hline Allowed (\%) & 3.11 \\
\hline Outliers (\%) & 0.00 \\
\hline Rotamer outliers (\%) & 0.52 \\
\hline Clashscore & 5.00 \\
\hline Average B-factor & 35.99 \\
\hline Macromolecules & 35.29 \\
\hline Ligands & 40.19 \\
\hline Solvent & 42.07 \\
\hline
\end{tabular}

* Values for the highest-resolution shell are shown in parentheses. 
A

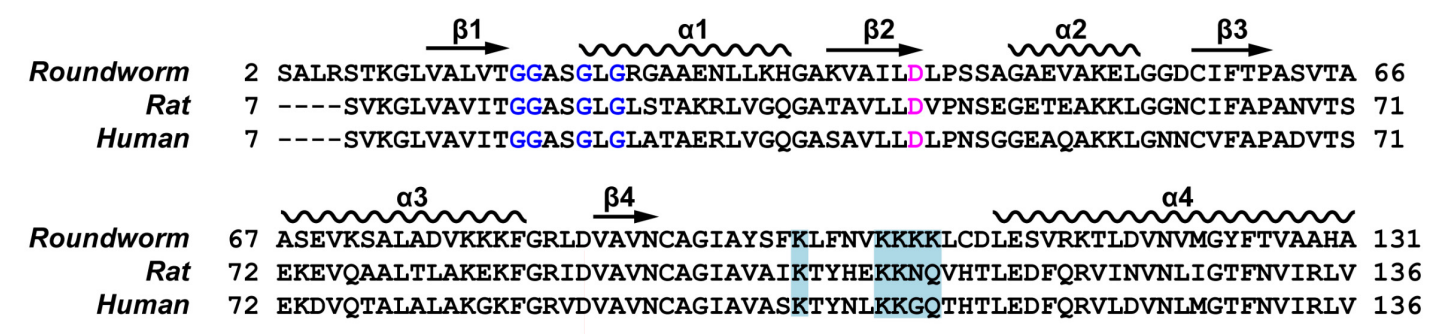

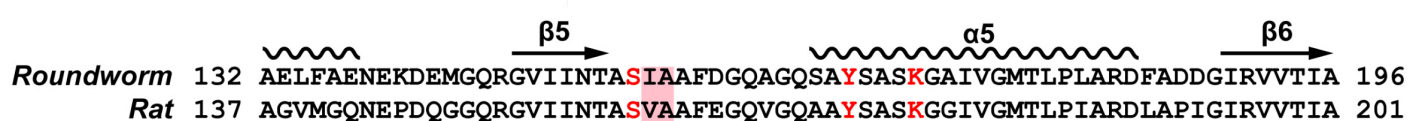

Human 137 AGEMGQNEPDQGGQRGVIINTASVAAFEGQVGQAAYSASKGGIVGMTLPIARDLAPIGIRVMTIA 201

$\begin{array}{rlll}\text { Roundworm } & 197 & \text { PGIFDTPMMASFPDKVRNFLIGLVPNPKRFGVPEEYGALVRHIIENRYLNGEVIRLDGALRMPA } & 260 \\ \text { Rat } & 202 & \text { PGLFATPLLTTLPDKVRNFLASQVPFPSRLGDPAEYAHLVQMVIENPFLNGEVIRLDGAIRMQP } & 265 \\ \text { Human } & 202 & \text { PGLFGTPLLTSLPEKVRNFLASQVPFPSRLGDPAEYAHLVQAIIENPFLNGEVIRLDGAIRMQP } & 265\end{array}$

B

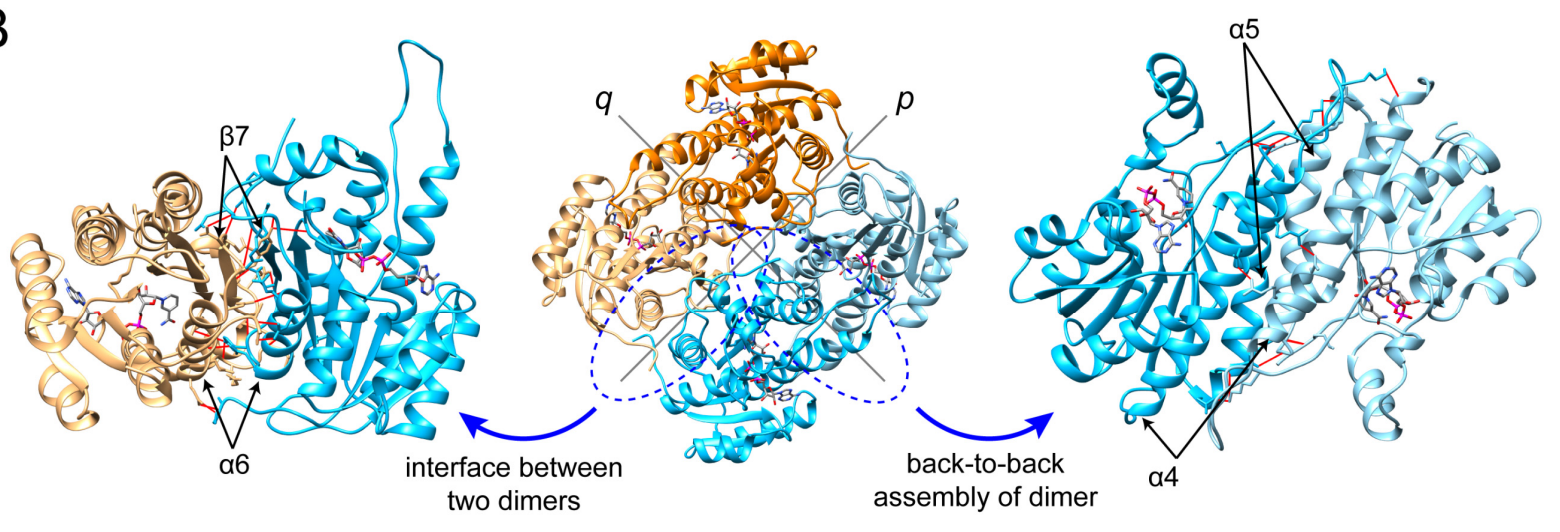

Figure S1. Crystal structure of AsHadh2. (A) Sequence alignment of AsHadh2, rHadh2, and hHadh2. Secondary structure elements are drawn based on the AsHadh2 structure. Catalytic residues (red), D40 involved in $\mathrm{NAD}^{+}$selectivity (magenta), and conserved GGXXGXG Rossmann motif for dinucleotide binding (blue) are highlighted in color. Residues proposed to be involved in acyl-CoA binding are shaded in a box ( $\alpha$-methyl group binding pocket, pink; diphosphate linkage, light blue). (AsHadh2, A. suum; rHadh2, Rattus norvegicus; hHadh2, Homo sapiens) (B) The dimer interface forming the back-to-back assembly and the interface between two dimers forming the tetramer. Structure of the tetrameric AsHadh2 assembly as a ribbon diagram with $\mathrm{NAD}^{+}$(stick) showing the location of the active site. Back-to-back dimers are shown in blue and orange. Within the dimer, each monomer unit is shaded in dark or light. The two dimers are related by a $p$ axis of symmetry, whereas the two subunits in the dimers are related by a $q$ axis of symmetry. Hydrogen bonds between two monomers are indicated by solid red lines. The key secondary structure elements involved in the interface are labelled. 


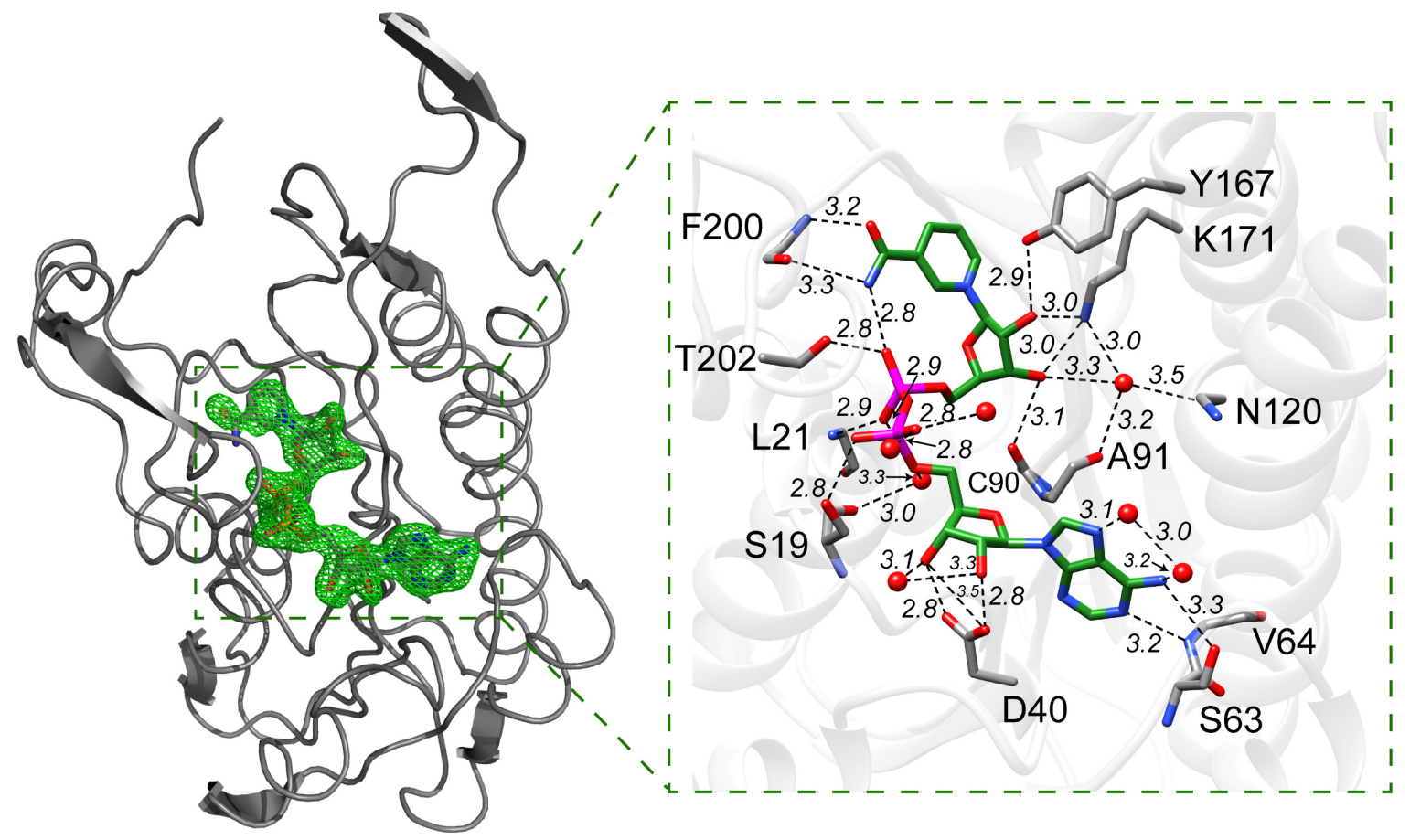

Figure S2. AsHadh2 bound to $\mathrm{NAD}^{+}$. The $F_{o}-F_{c}$ omit map for the ligand $\mathrm{NAD}^{+}$(green) was calculated by removing ligands before refinement and is contoured at $\pm 3 \sigma$. The bound $\mathrm{NAD}^{+}$ cofactor is shown in stick with carbons colored in green. Dashed lines indicate hydrogen bond contacts with distances $(\AA)$ between two atoms noted. Side chains or main chains interacting with the $\mathrm{NAD}^{+}$are show in stick with carbons colored in gray. Structured waters are shown as red spheres. (O, red; $\mathrm{N}$, blue; $\mathrm{P}$, fuchsia) 


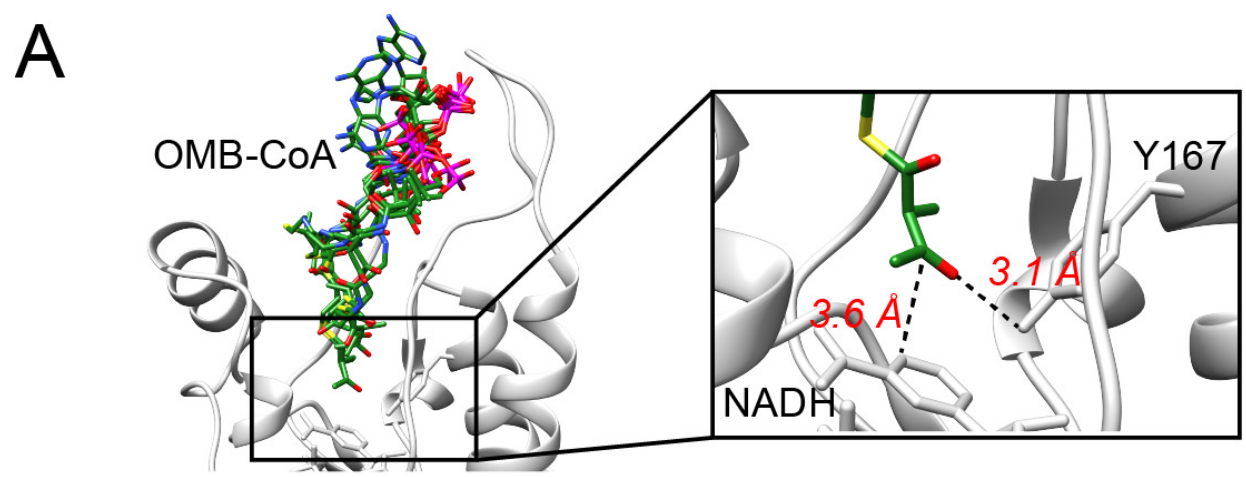

B
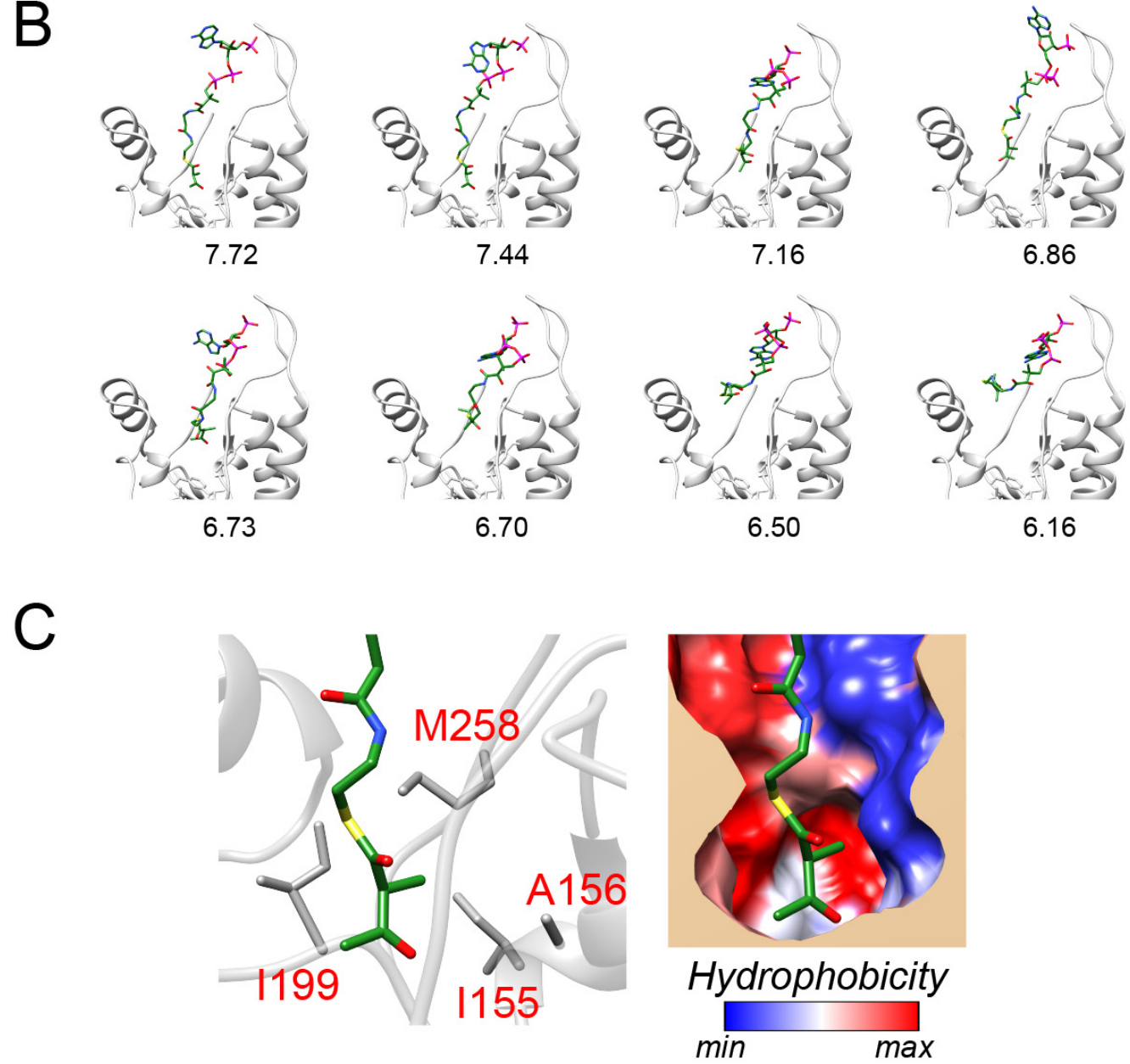

Figure S3. Docking of (S)-3-oxo-2-mehthylbutyryl-CoA into AsHadh2. (A) Eight given poses of ligand are shown here of the OMB-CoA (C, green) docked into the AsHadh2-NADH crystal structure (left) with a zoomed in view of the pose with highest docking score (right). (B) The eight poses shown overlaid in Figure S3A with scores. (C) Structural features involved in binding $\alpha$-methyl group of substrate. A surface representation of the hydrophobic pocket formed by I155, A156, I199, and M258 is shown colored by hydrophobicity. The key residues for the hydrophobic interaction with $\alpha$-methyl group of OMB-CoA (C, green) are show in stick model. (C, grey; O, red; N, blue; S, yellow; P, fuchsia) 
A

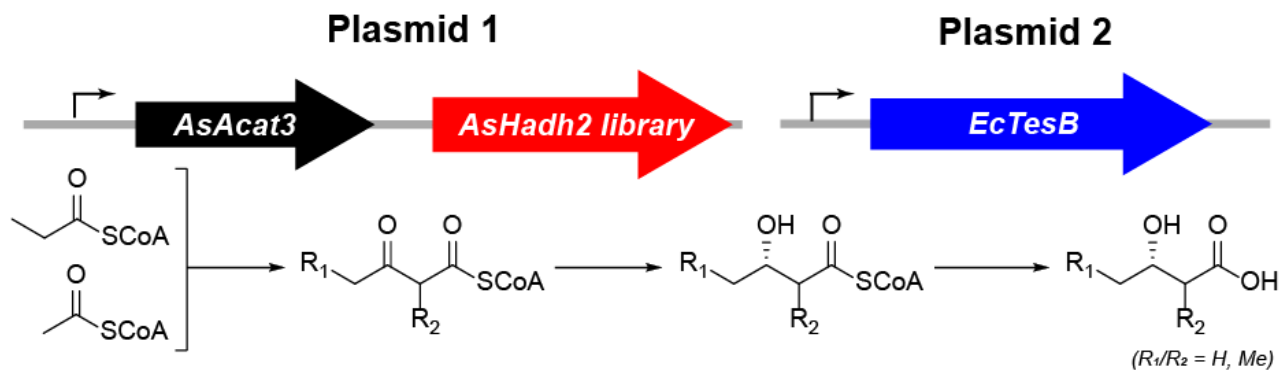

B

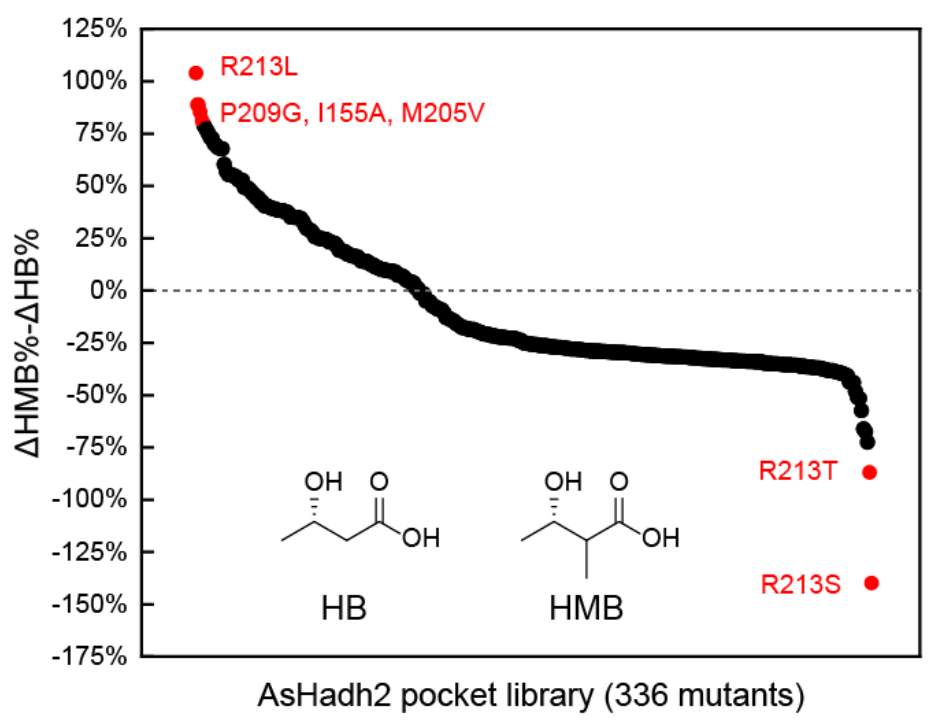

\begin{tabular}{cc}
\hline Mutations & $\Delta H M B \%-\Delta H B \%$ \\
\hline R213L & $104 \%$ \\
P209G & $85 \%$ \\
I155A & $79 \%$ \\
P209G & $77 \%$ \\
M205V & $75 \%$ \\
R213P & $-68 \%$ \\
R213T & $-72 \%$ \\
R213T & $-87 \%$ \\
R213S & $-140 \%$ \\
\hline
\end{tabular}

Figure S4. Generation and screening of an AsHadh2 pocket library. The random mutation of these pocket-residues was performed using NNK degenerate codon mutagenesis to form 14 AsHadh2 libraries (A94, A153, I155, A156, Q164, P197, G198, I199, F200, M204, M205, F208, P209, and R213L). 24 colonies were picked from each positional library for preliminary screening of 336 total variants. (A) AsHadh2 variants were analyzed using an in vivo screen in E. coli bearing a pathway for the production of 3-hydroxy acids. (B) E. coli BAP1 was transformed with pTrc33-TesB pAsAcat3-AsHadh2*. After $2 \mathrm{~d}$, cell cultures were analyzed for production of branched (HMB) vs linear (HB) product. Data are plotted in terms of the sum of the percent increase in HMB and percent decrease in HB compared to wild-type. (AsAcat3, thiolase from A. suum, AsHadh2, variant from library; TesB, thioesterase from E. coli). Based on these initial results, positions I155, M205, P209 and R213 (red) were selected for more detailed analysis. 


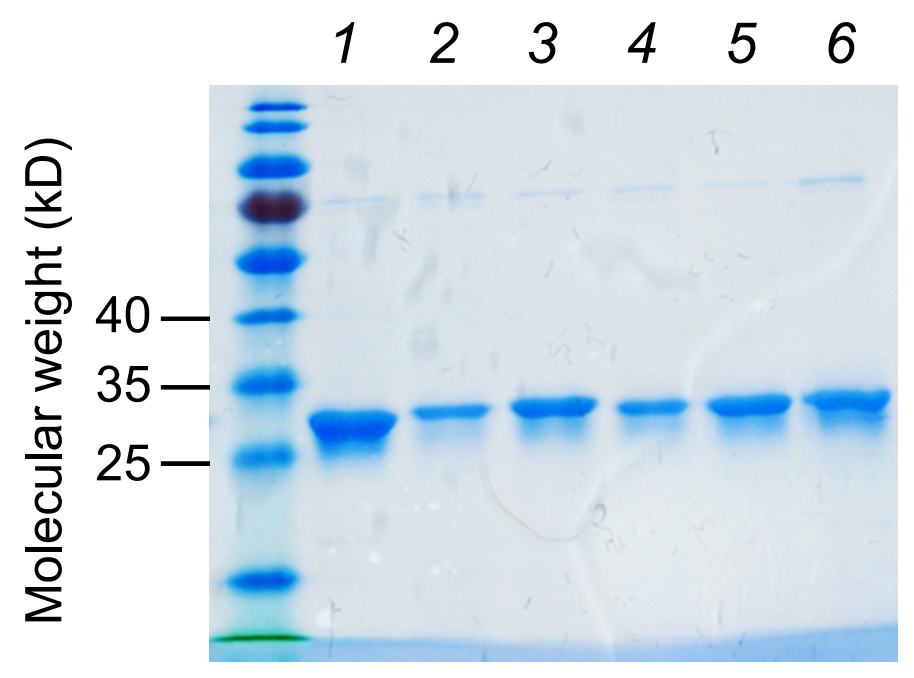

Figure S5. SDS-PAGE of purified Hadh2 variants. Lane 1, wild type; lane 2, I155A; lane 3, I155T; lane 4, M205F; lane 5, Hadh2, R213S; lane 6, R213L. 
A

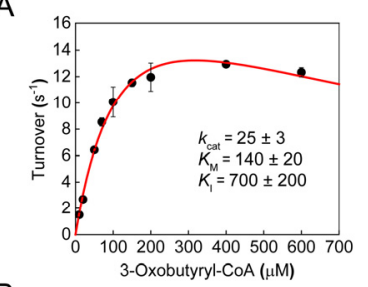

B

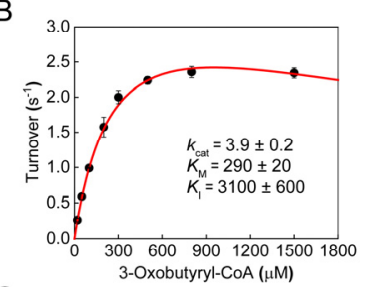

C

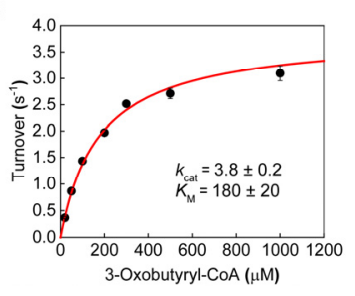

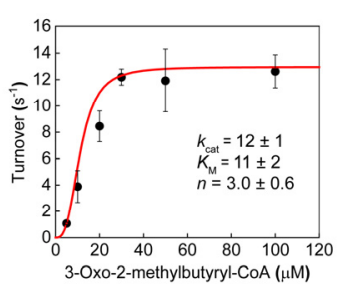
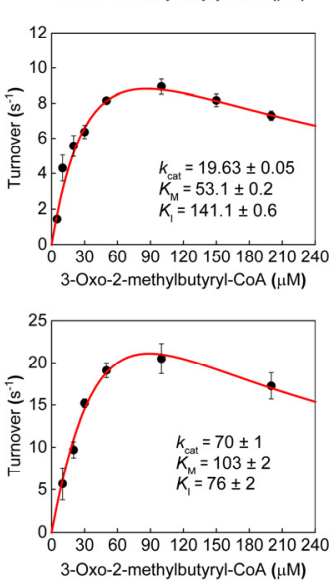

D

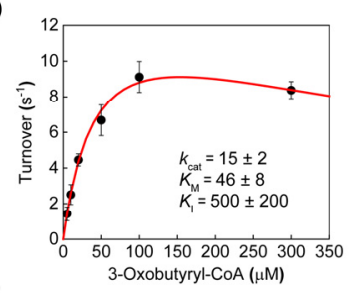

E

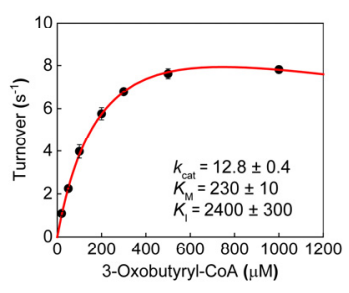

$\mathrm{F}$

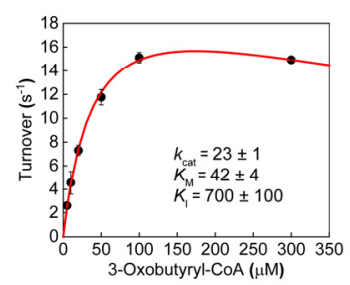

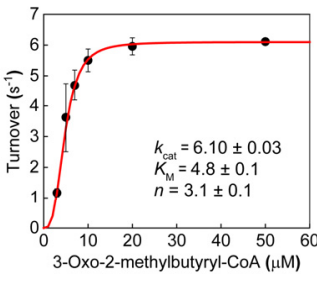
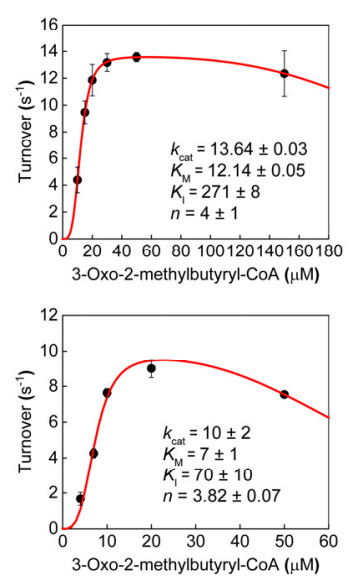

Figure S6. Steady-state kinetic analysis for Hadh2 variants with OMB-CoA and OB-CoA. Initial rate data for Hadh2 variants for the reduction of branched (OMB-CoA) or linear (OBCoA) were collected as a function of substrate concentration by monitoring oxidation of NADH. Data were fit to the standard Michaelis-Menten equation (Eq. 1), the Michaelis-Menten equation with substrate inhibition (Eq. 2), the Hill equation (Eq. 3), or the Hill equation with substrate inhibition (Eq. 4) as noted in the method. Data are mean \pm standard error $(n=3)$. (A) Wildtype AsHadh2. (B) AsHadh2 I155A. (C) AsHadh2 I155T. (D) AsHadh2 M205F. (E) AsHadh2 R213L. (F) AsHadh2 R213S. 
A

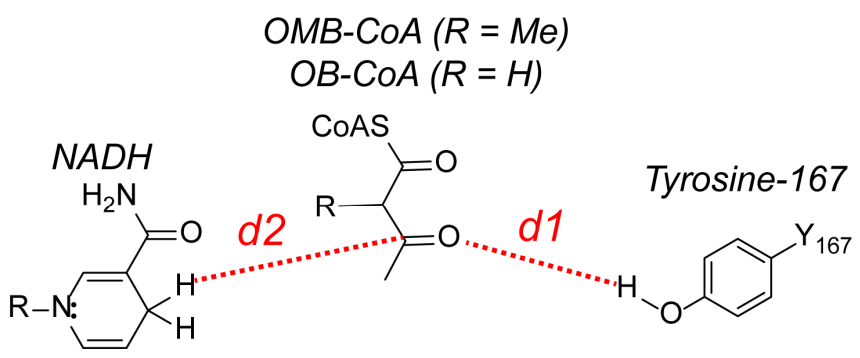

B
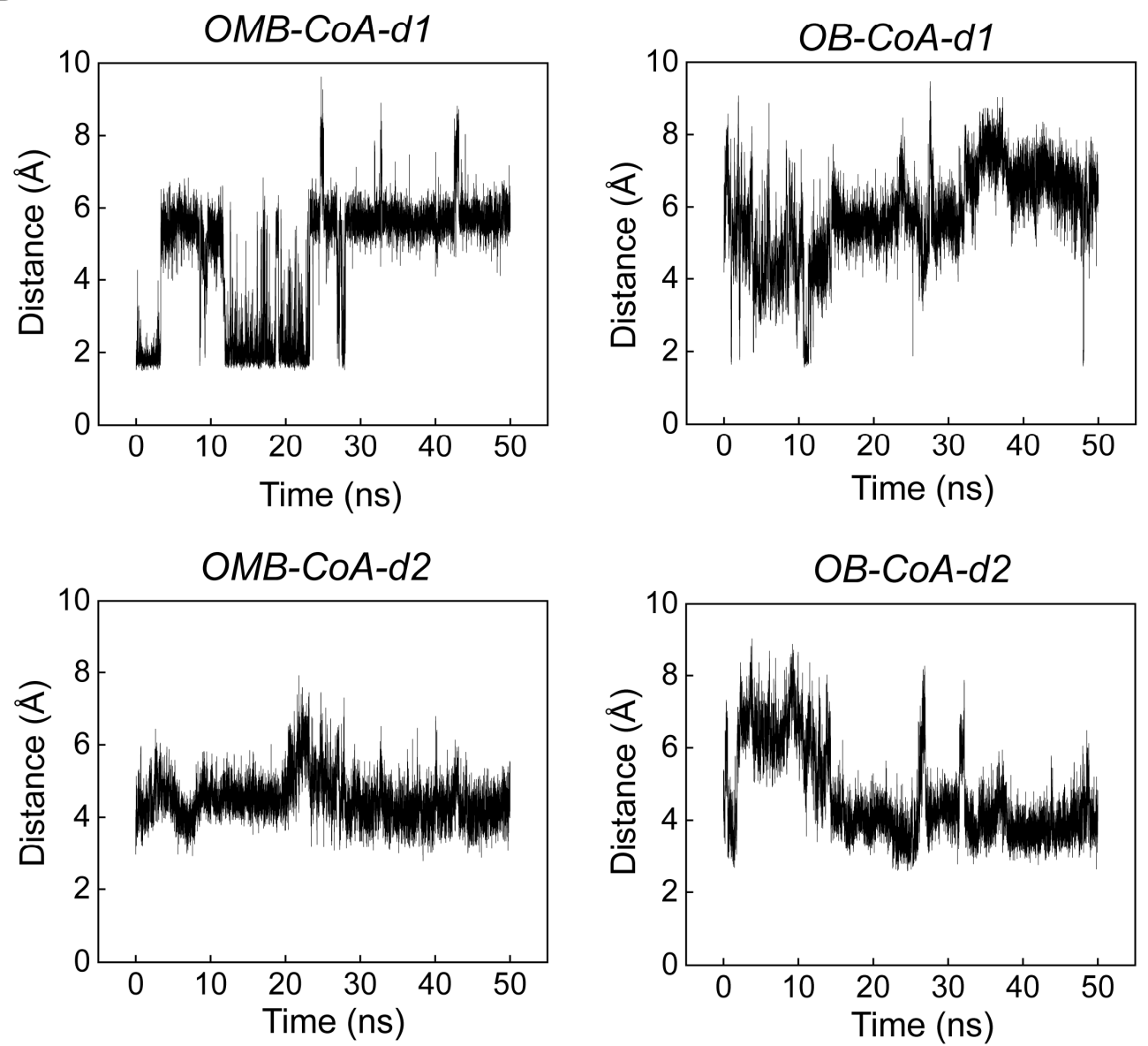

Figure S7. Molecular dynamics simulations of AsHadh2. The structure of AsHadh2-NAD ${ }^{+}$ with either OMB-CoA (branched) or OB-CoA (unbranched) docked as a ligand was used for molecular dynamics simulations. The distances between the keto group and either Y167 $(d l)$ or NADH $(d 2)$ were plotted to monitor the distances of the reacting centers as they are considered to serve as a minimal metric for monitoring formation of an 'active conformation' for hydride transfer $[13,14]$. (A) Distances between reacting centers where $d 1$ is $\mathrm{O} 3 \mathrm{O}(\mathrm{M}) \mathrm{BCoA}-\mathrm{HHY} 167$ and $d 2$ is $\mathrm{C} 3 \mathrm{O}(\mathrm{M}) \mathrm{BCoA}-\mathrm{H} 4 \mathrm{~N} 1 \mathrm{NADH}$. (B) The profiles of $d 1$ and $d 2$ for ligands OMB-CoA and OB-CoA in $50 \mathrm{~ns}$ simulation process. The active conformation was defined as $d 1 \leq 3.4 \AA$ and $d 2 \leq 4.5 \AA$ [13]. The proportions of pre-reaction conformations of AsHadh2 were $11.8 \%$ for OMB-CoA, and $0.4 \%$ for OB-CoA. 


\section{References}

1. Gibson, D. G.; Young, L.; Chuang, R.-Y.; Venter, J. C.; Hutchison, C. A.; Smith, H. O., Enzymatic assembly of DNA molecules up to several hundred kilobases. Nat. Protoc. 2009, 343-345.

2. Dong, H.; Liffland, S.; Hillmyer, M. A.; Chang, M. C., Engineering in vivo production of $\alpha$-branched polyesters. J. Am. Chem. Soc. 2019, 141, 16877-16883.

3. Kabsch, W. XDS. Acta Crystallogr. D. Biol. Crystallogr. 2010, 66, 125-132.

4. Evans, P. R.; Murshudov, G. N. How good are my data and what is the resolution? Acta Crystallogr. D. Biol. Crystallogr. 2013, 69, 1204-1214.

5. Long, F.; Vagin, A. A.; Young, P.; Murshudov, G. N. BALBES: A molecular-replacement pipeline. Acta Cryst. D. Biol. Crystallogr. 2008, 64, 125-132.

6. Emsley, P.; Lohkamp, B.; Scott, W. G.; Cowtan, K. Features and development of Coot. Acta Crystallogr. D Biol. Crystallogr. 2010, 66, 486-501.

7. Adams, P. D.; Afonine, P. V.; Bunkóczi, G.; Chen, V. B.; Davis, I. W.; Echols, N.; Headd, J. J.; Hung, L.-W.; Kapral, G. J.; Grosse-Kunstleve, R. W.; McCoy, A. J.; Moriarty, N. W.; Oeffner, R.; Read, R. J.; Richardson, D. C.; Richardson, J. S.; Terwilliger, T. C.; Zwart, P. H. PHENIX: a comprehensive Python-based system for macromolecular structure solution. Acta Crystallogr. D. Biol. Crystallogr. 2010, 66, 213-221.

8. Pettersen, E. F.; Goddard, T. D.; Huang, C. C.; Couch, G. S.; Greenblatt, D. M.; Meng, E. C.; Ferrin, T. E. UCSF Chimera: A visualization system for exploratory research and analysis. J. Comput. Chem. 2004, 25, 1605-1612.

9. Friesner, R. A.; Banks, J. L.; Murphy, R. B.; Halgren, T. A.; Klicic, J. J.; Mainz, D. T.; Repasky, M. P.; Knoll, E. H.; Shelley, M.; Perry, J. K.; Shaw, D. E.; Francis, P.; Shenkin, P. S., Glide: A new approach for rapid, accurate docking and scoring. 1. Method and assessment of docking accuracy. J. Med. Chem. 2004, 47, 1739-1749.

10. Halgren, T. A.; Murphy, R. B.; Friesner, R. A.; Beard, H. S.; Frye, L. L.; Pollard, W. T.; Banks, J. L., Glide: a new approach for rapid, accurate docking and scoring. 2. Enrichment factors in database screening. J. Med. Chem. 2004, 47, 1750-179.

11. Pfeifer, B. A.; Admiraal, S. J.; Gramajo, H.; Cane, D. E.; Khosla, C., Biosynthesis of complex polyketides in a metabolically engineered strain of E. coli. Science 2001, 291, 1790-1792.

12. Blaisse, M. R.; Dong, H.; Fu, B.; Chang, M. C., Discovery and engineering of pathways for production of $\alpha$-branched organic acids. J. Am. Chem. Soc. 2017, 139, 14526-14532.

13. Zhou, J.; Wa,ng, Y.; Xu, G.; Wu, L.; Han, R.; Schwaneberg, U.; Rao, Y.; Zhao, Y. L.; Zhou, J.; Ni Y. Structural insight into enantioselective inversion of an alcohol dehydrogenase reveals a "Polar Gate" in stereorecognition of diaryl ketones. J. Am. Chem. Soc. 2018, 140, 12645-12654. 
14. Mugnai , M. L.; Shi, Y.; Keatinge-Clay, A. T.; Elber, R. Molecular dynamics studies of modular polyketide synthase ketoreductase stereospecificity. Biochemistry 2015, 54, 2346-2359. 